\title{
Dinamometria de preensão manual como parâmetro de avaliação funcional do membro superior de pacientes hemiparéticos por acidente vascular cerebral
}

\author{
Handgrip dynamometry as a parameter of functional evaluation of the upper extremity \\ bemiparetic after stroke
}

Antonio Vinicius Soares ${ }^{1}$, Caroline Kerscher ${ }^{2}$, Loisiane Uhlig ${ }^{2}$, Susana Cristina Domenech ${ }^{3}$, Noé Gomes Borges Júnior ${ }^{3}$

Estudo desenvolvido na Faculdade Guilherme Guimbala da

Associação Catarinense de Ensino (FGG-ACE) - Joinville (SC), Brasil.

${ }^{1}$ Fisioterapeuta; Mestre em

Ciências do Movimento Humano, Pesquisador do Núcleo de Pesquisas em Neuroreabilitação (NUPEN) do Curso de Fisioterapia da FGG-ACE Joinville (SC), Brasil.

${ }^{2}$ Acadêmica do Curso de Fisioterapia da FGG-ACE - Joinville (SC), Brasil.

${ }^{3}$ Professor Doutor do Programa de Pós-graduação em Ciências do Movimento Humano do Centro de Ciências da Saúde e do Esporte (CEFID) da Universidade do Estado de Santa Catarina (UDESC) Florianópolis (SC), Brasil.

\section{ENDEREÇO PARA CORRESPONDÊNCIA}

Antonio Vinicius Soares - Núcleo de Pesquisas em Neuroreabilitação (NUPEN) do Curso de Fisioterapia da Faculdade Guilherme Guimbala da Associação Catarinense de Ensino (FGG-ACE) - Rua São José, 490 - Centro - CEP: 89202-010

- Joinville (SC), Brasil - E-mail: a.vini@ig.com.br

\section{APRESENTACSÃO} fev. 2011

ACEITO PARA PUBLICAÇÃO set. 2011

\section{FONTE DE FINANCIAMENTO} nenhuma

\section{CONFLITO DE INTERESSE}

nada a declarar

Parecer de aprovação do Comitê de Ética em Pesquisa da Associação Educacional Luterana Bom Jesus/ IELUSC $n^{\circ} 006-2010$
RESUM0: A força muscular é a valência física mais importante. É provável que exista correlação entre a força de preensão manual e outros testes para o membro superior afetado por um acidente vascular cerebral (AVC). O propósito deste estudo é analisar o valor preditivo da dinamometria de preensão manual (DPM) para recuperação do membro superior parético por AVC. Foram avaliados 43 pacientes hemiparéticos pós-AVC $(60,7$ anos $\pm 12,1)$. Vários testes (Escala de movimento da mão (EMM), Estesiometria, Teste de caixa e blocos, 9 buracos e pinos, Escala de Ashworth modificada e Índice de Barthel) foram relacionados com a DPM. Os resultados apontaram que a DPM apresenta boa correlação com EMM e, contrariamente, os testes de destreza manual, a sensibilidade e o índice de independência funcional não apresentaram valores significativos. A DPM é um teste rápido, fácil e acessível, e pode fazer parte dos protocolos de avaliação funcional do membro superior de pacientes hemiparéticos por AVC.

DEscRITORES: acidente vascular cerebral; paresia.

ABSTRACT: Muscle strength is the most important physical valence. It is likely that there is a correlation between handgrip strength and other tests for the upper extremity affected by stroke. The purpose of this study is to analyze the predictive value of handgrip dynamometry (HD) for upper limb recovery in stroke. Were studied 43 hemiparetic patients after stroke (60.7 years \pm 12.1$)$. Several tests (Hand moviment scale (HMS), Esthesiometry, Box and blocks test, 9 hole and peg test, Modified Ashworth scale and Barthel index) were related to HD. The results showed that there is good correlation of the HD with HMS and, by contrast, the tests of manual dexterity, the sensitivity and index of functional independence showed no significant values. The HD is a quick, easy and affordable test, and can be part of protocols for functional evaluation of upper extremity of hemiparetic patients by stroke.

KeYwords: stroke; paresis. 


\section{INTRODUCÃO}

O acidente vascular cerebral (AVC) é um importante problema de saúde pública, caracterizado por um distúrbio na função cerebral, de origem vascular. Ele é uma das quatro principais causas de morte em muitos países, e é responsável por grande número de pacientes com sequelas neurológicas ${ }^{1,2}$. Dentre os distúrbios gerados, a hemiparesia é um dos comprometimentos mais evidentes e se refere à fraqueza muscular em um hemicorpo como consequência da lesão no encéfalo ${ }^{3}$.

Atualmente, o membro superior de pacientes hemiparéticos por AVC é um elemento de valorização para independência funcional e vem recebendo mais atenção e cuidados dentro do processo reabilitacional. Para tanto, sua avaliação é de extrema importância, tendo em vista que possibilita qualificar e quantificar as sequelas após o $\mathrm{AVC}^{1,2}$. É por meio dela que o tratamento pode ser traçado com foco exclusivo aos danos deixados pelo evento ${ }^{1}$.

A seleção de instrumentos de medida apropriados durante o processo de avaliação permite traçar o real perfil de cada paciente. As informações obtidas norteiam o plano de tratamento e permitem estabelecer metas realísticas quanto ao prognóstico. Dessa forma, expectativas de recuperação funcional por parte dos familiares e do próprio paciente podem ser discutidas com mais clareza e confiabilidade.

O propósito deste estudo é analisar o valor preditivo de diferentes testes clínicos para recuperação do membro superior parético por AVC, correlacionando a dinamometria de preensão manual com a motricidade manual, sensibilidade, destreza, tônus muscular e independência funcional.

\section{METODOLOGIA}

Foi realizado um estudo descritivo do tipo correlacional. Participaram 43 pacientes que sofreram AVC, com quadro de hemiparesia, sendo 18 mulheres $(41,86 \%)$ e 25 homens (58,13\%) com idade média de 60,7 anos $( \pm 12,1)$ e tempo médio de lesão de 22 meses $( \pm 25,1)$. Destes, 39,5\% apresentavam hemiparesia à direita e $60,5 \%$ à esquerda. Como critério de inclusão, os pacientes deveriam registrar quadro de hemiparesia por AVC, com função ao menos parcial do membro acometido (capaz de realizar o alcance e preensão), independente da idade e sexo. Os critérios estabelecidos para exclusão foram qualquer outra causa de hemiparesia, déficits visuais e/ou auditivos graves, déficits cognitivos e afasia.

A análise foi realizada no ambulatório de Reabilitação Neurológica do Curso de Fisioterapia da Faculdade Guilherme Guimbala da Associação Catarinense de Ensino (FGG/ACE) e na Associação de Deficientes Físicos de Joinville (ADEJ).

Este estudo foi aprovado pelo Comitê de Ética em Pesquisa da Associação Educacional Luterana Bom Jesus/IELUSC ( $n^{\circ}$ 006-2010). Os objetivos e os procedimentos foram explicados aos participantes que, ao concordarem com a participação voluntária, assinaram um termo de consentimento livre e esclarecido.

Para maior confiabilidade da pesquisa, os testes foram realizados sempre pelo mesmo examinador. Uma ficha com dados pessoais e anamnese foi preenchida. Todas as avaliações foram realizadas primeiramente com o membro não acometido, seguindo-se do membro parético. Os instrumentos utilizados na avaliação podem ser visualizados na Figura 1.

Para avaliação da força muscular, utilizou-se o dinamômetro de preensão manual TKK 5401 GRIP-D ${ }^{\circledR}$ TAKEI Scientific Instruments - Japan (capacidade de mensuração de 5 a 100 kg; resolução 0,05 kg; precisão $\pm 0,5 \%$ ). No momento dos testes, o paciente estava posicionado sentado confortavelmente, com o ombro aduzido, cotovelo fletido a $90^{\circ}$, antebraço e punho em posição neutra ${ }^{4-6}$. Quatro mensurações eram realizadas em cada membro, sendo a primeira utilizada para adaptação e conhecimento do equipamento e, por conta disso, descartada. Com as demais medidas calculou-se a média aritmética.

Para o teste de escala de movimento da mão (pontuação de 1 a 6), o paciente realizava extensão seguida de flexão dos dedos; extensão apenas do segundo dedo, como se estivesse apontando; e pinça entre o primeiro e os demais dedos até chegar à oponência com o dedo mínimo ${ }^{6,7}$.

$\mathrm{Na}$ avaliação do tônus muscular por meio da Escala de Ashworth modificada, foram selecionados os músculos flexores de cotovelo, punho e dedos. Obteve-se o resultado calculando a média do grau de espasticidade do membro como um todo. Para facilitar a tabulação e cálculo dos dados, usou-se a escala de 0 a $5^{8,9}$.

Já na avaliação da destreza manual foram utilizados os testes de nove buracos e pinos (Nine hole peg test-9HPT), composto por nove pinos $(9 \mathrm{~mm}$ de diâmetro e $32 \mathrm{~mm}$ de comprimento), uma prancha de madeira com dimensão de 100x100x20 mm contendo nove buracos de $10 \mathrm{~mm}$ de diâmetro e $15 \mathrm{~mm}$ de profundidade ${ }^{10,11}$. O paciente era orientado a retirar todos os pinos do tablado e, em seguida, colocá-los novamente. Permitiu-se 15 segundos para o primeiro contato e conhecimento do equipamento, e então o tempo total para completar a tarefa era cronometrado, não podendo ultrapassar 300 segundos para o fim. Outro teste aplicado foi o de caixa e blocos (Box and blocks test - BBT), que compreende uma caixa

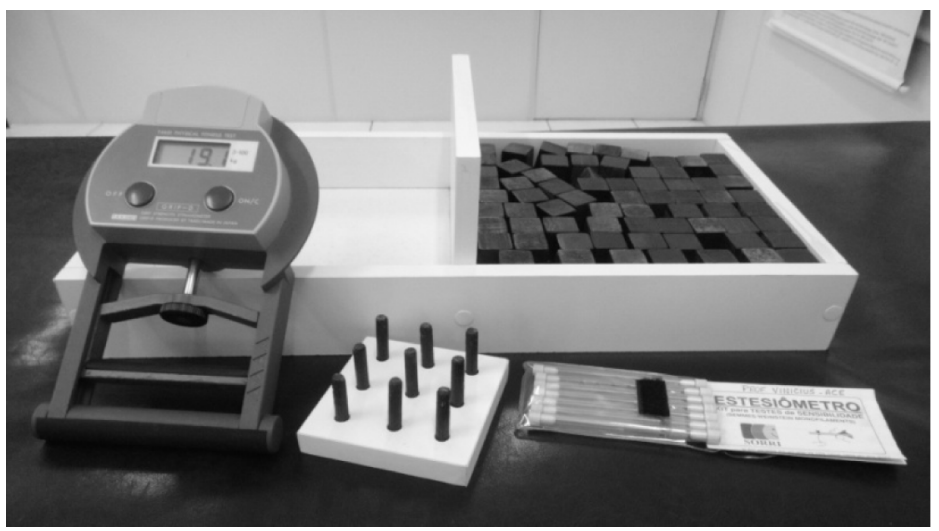

Figura 1. Instrumentos utilizados para avaliação 
de madeira (53,7 cm de comprimento) com uma divisória mais alta que as suas bordas, separando-a em dois compartimentos de iguais dimensões e 150 cubos de $2,5 \mathrm{~cm}^{10,11}$. O paciente transportava os blocos de um lado a outro da caixa, sendo o critério dos 15 segundos iniciais de adaptação também utilizado. $\mathrm{Na}$ sequência, um minuto foi cronometrado e contou-se o número de blocos transportados. Para ambos os testes houve comando verbal, instruindo o participante a realizar a tarefa o mais rápido possível.

Avaliou-se a sensibilidade por Estesiometria, utilizando um conjunto de seis monofilamentos de nylon (Monofilamentos Sorri-Baurú ${ }^{\circledR}$ ) - eles exerceram força específica na área testada que corresponde à variação de peso de 0,05 a 300,00 g. Os monofilamentos foram aplicados perpendicularmente à pele, conforme o mapa de distribuição dos nervos ulnar, mediano e radial, em pontos determinados pelo protocolo do teste. Com os olhos vendados, o paciente relatava a percepção do toque e o localizava ${ }^{12,13}$.

Utilizou-se o Índice de Barthel para análise da independência funcional do indivíduo. Aplicado ao paciente e/ou acompanhante, continha questões sobre alimentação, higiene pessoal e vestuário, entre outras. Seu escore máximo foi de 100 pontos, sendo este o valor sugestivo de independência total ${ }^{14}$.

Os dados coletados foram tabulados no Microsoft Office Excel ${ }^{\circledR} 2007$, no qual foram calculadas as médias e os desvios padrões. Para a análise de regressão linear múltipla e o cálculo de correlação momento de Pearson utilizou-se o software GraphPad Prism $4{ }^{\circledR}$.

\section{RESULTADOS}

Como referência para análise dos dados desta pesquisa, usou-se a dinamometria de preensão manual (DPM), por ser apontada como um instrumento de medida de fácil e rápida aplicação, além de estar bem documentado na literatura ${ }^{4-6}$. Assim, cada um dos outros instrumentos utilizados foram correlacionados individual e simultaneamente com a DPM.
Os resultados na sequência se referem aos valores da correlação momento de Pearson e análise da regressão linear múltipla aplicados sobre os dados coletados na pesquisa.

As Tabelas 1 e 2 apresentam os coeficientes de correlação encontrados, assim como os níveis de significância destas correlações.

Nas Tabelas 3 e 4 as mesmas análises são apresentadas para os pacientes espásticos, que representam $69,8 \%$ do total da amostra.

Em geral, os coeficientes de correlação (positivos ou negativos) considerados

Tabela 1. Correlação da dinamometria de preensão manual com os demais testes (todos os pacientes)

\begin{tabular}{lccccc}
\hline & CB & 9BP & EMM & ESTESIO & BARTHEL \\
\hline Pearson $(r)$ & 0,4060 & $-0,4740$ & 0,4660 & $-0,1350$ & 0,1680 \\
Valor $p$ & $0,0069^{*}$ & $0,0013^{*}$ & $0,0016^{*}$ & 0,3866 & 0,2828 \\
\hline
\end{tabular}

*Nível de significância p<0,05; CB: Teste de caixa e blocos; 9BP: Teste dos nove buracos e pinos; EMM: Escala de movimentos da mão; Estesio: Estesiometria; Barthel: Índice de Barthel

Tabela 2. Regressão linear múltipla da dinamometria de preensão manual com os demais testes (todos os pacientes)

\begin{tabular}{lccccc}
\hline & CB & $9 B P$ & EMM & ESTESIO & BARTHEL \\
\hline$r^{2}$ & 0,1648 & 0,2248 & 0,2175 & 0,0183 & 0,0280 \\
Valor $\mathrm{p}$ & $0,0069^{*}$ & $0,0013^{*}$ & $0,0016^{*}$ & 0,3866 & 0,2828 \\
\hline
\end{tabular}

*Nível de significância p<0,05; CB: Teste de caixa e blocos; 9BP: Teste dos nove buracos e pinos; EMM: Escala de movimentos da mão; Estesio: Estesiometria; Barthel: Índice de Barthel; r: Pearson

Tabela 3. Correlação da dinamometria de preensão manual com os demais testes (somente pacientes espásticos)

\begin{tabular}{|c|c|c|c|c|c|c|}
\hline & CB & $9 B P$ & EMM & ESTESIO & BARTHEL & ASHWORTH \\
\hline Pearson (r) & 0,5353 & $-0,5525$ & 0,4804 & $-0,1760$ & 0,3802 & $-0,2351$ \\
\hline Valor $p$ & $0,0023^{*}$ & $0,0015^{*}$ & $0,0072 *$ & 0,3522 & $0,0382^{*}$ & 0,2110 \\
\hline
\end{tabular}

*Nível de significância $\mathrm{p}<0,05$; CB: Teste de caixa e blocos; 9BP: Teste dos nove buracos e pinos; EMM: Escala de movimentos da mão; Estesio: Estesiometria; Barthel: Índice de Barthel; Ashworth: Escala de Ashworth modificada

Tabela 4. Regressão linear múltipla da dinamometria de preensão manual com os demais testes (somente pacientes espásticos)

\begin{tabular}{|c|c|c|c|c|c|c|}
\hline & CB & $9 B P$ & EMM & ESTESIO & BARTHEL & ASHWORTH \\
\hline Pearson (r) & 0,2866 & 0,3053 & 0,2308 & 0,8951 & 0,1446 & 0,0553 \\
\hline Valor $p$ & $0,0023^{*}$ & $0,0015^{*}$ & $0,0072 *$ & 0,3522 & $0,0382 *$ & 0,2110 \\
\hline
\end{tabular}

*Nível de significância p<0,05; CB: Teste de caixa e blocos; 9BP: Teste dos nove buracos e pinos; EMM: Escala de movimentos da mão; Estesio: Estesiometria; Barthel: Índice de Barthel; Ashworth: Escala de Ashworth modificada 
significativos apresentaram-se moderados a fortes. Obteve-se correlação positiva para a Escala de movimento da mão (EMM) e o teste da caixa e blocos, levando a crer que maiores medidas de dinamometria correspondem a melhor desempenho nos testes de destreza do membro superior. Quanto ao teste de nove buracos e pinos, a correlação foi negativa, representando que com maior força de preensão manual, menor é o tempo de execução no teste. Já o de Ashworth indicou que resultados meIhores na motricidade manual (força e destreza) sugerem menor grau de espasticidade.

Os pacientes envolvidos na pesquisa apresentavam espasticidade classificada como leve $(0,7$ na escala de 0,0 a 5,0). A sensibilidade (Estesio) e a independência funcional (Barthel) não demonstraram correlação com a motricidade da mão, pois apresentaram nível de significância $>0,05$. Já nos pacientes espásticos, houve correlação significativa quanto à independência funcional.

\section{DISCUSSÃO}

Há tempos os pesquisadores se interessam por aprimorar a avaliação inicial e as diferentes fases da evolução de sobreviventes de $\mathrm{AVC}^{6,15-17}$. A criteriosa e precisa seleção dos instrumentos de medida permitem traçar diretrizes claras de tratamento, além do possível estabelecimento de um prognóstico de recuperação motora e funcional desses pacientes $^{6,18,19}$.

Após o AVC, $80 \%$ dos sobreviventes apresentam paresia aguda do membro superior, e somente um terço deles alcança recuperação funcional ${ }^{19}$.

Kwakkel et al. ${ }^{19}$ estudaram o impacto do tempo sobre a recuperação em 101 pacientes hemiparéticos por AVC. Os pesquisadores utilizaram o Índice de Barthel, Escala de Fugl-Meyer, avaliação da marcha e atenção visuo-espacial. Eles demonstraram que $16 \%$ da recuperação inicial é espontânea (6 a 10 semanas). Observaram ainda que a melhor recuperação ocorre nos primeiros três meses. Porém, ao contrário desses achados, outras pesquisas argumentam que a recuperação contínua é dependente de vários outros fatores de difícil controle $\mathrm{e}^{20-22}$.
Nesta pesquisa, a força de preensão manual (DIN) apresentou boa correlação com a motricidade manual. Isso é relevante, pois a dinamometria é um procedimento rápido, seguro, não exige treinamento extensivo e o instrumento é acessível à maioria dos clínicos. De fato, a medida tem sido correlacionada fortemente como preditora da recuperação do membro superior, como observado no movimento de alcance ${ }^{23}$, e do membro superior como um todo ${ }^{6,7}$.

Assim, a dinamometria, bem como a EMM, demonstraram boa correlação com os testes de destreza manual. Como apresentado nos resultados, foram utilizados dois testes, o de nove buracos e pinos e o da caixa e blocos. Em geral, bons desempenhos nestes testes permitem estimar a funcionalidade do membro superior dos pacientes. Isso vem ao encontro com registros de outras pesquisas ${ }^{15,20,22-24}$.

A sensibilidade avaliada por Estesiometria não se correlacionou significativamente com a DPM ou outros instrumentos da avaliação. Os achados são congruentes com os encontrados por Zackowski et al. ${ }^{25}$, que também não encontraram boa correlação da sensibilidade com a força muscular do membro superior ou com a espasticidade. Rand et al. ${ }^{26}$ argumentam que a perda sensorial não afeta os ganhos funcionais no membro superior, porém tal afirmação é contestada quando o déficit sensitivo está associado a outros déficits, principalmente o motor ${ }^{27}$. Winward et al. ${ }^{28}$ encontraram grande variabilidade das alterações de sensibilidade em hemiparéticos por AVC. Assim, deve-se ter muita cautela na utilização isolada dos achados da sensibilidade, o que pode implicar em erros diagnósticos.

A independência funcional dos pacientes hemiparéticos foi avaliada pelo Índice de Barthel, que tem sido frequentemente usado para essa finalidade ${ }^{29,30}$. Não foi encontrada, no presente estudo, correlação significativa desse instrumento com a DPM, exceto quando analisados apenas os pacientes espásticos. Isso talvez tenha ocorrido por conta do perfil da amostra estudada, em que o escore médio foi alto $(87,4 / 100,0)$ e pela fase de recuperação em que se encontravam os pacientes - todos os casos eram crônicos (7-108 meses). Os resultados podem ter sido afetados, pois se sabe da recuperação espontânea nos primeiros meses, com consequente alteração nos escores do Índice de Barthel por conta das adaptações comportamentais, sem necessariamente haver recuperação motora e/ou funcional ${ }^{17}$.

Quanto à espasticidade, considerada leve no grupo de pacientes avaliados, não se observou correlação significativa com a DPM. Outras pesquisas observaram a influência da espasticidade com pior desempenho em testes de destreza manual do membro superior, bem como, a sua relação com a fraqueza muscular, em que pacientes mais espásticos apresentam maior déficit de força e destreza ${ }^{24,}$ Porém, deve-se ressaltar que, ao contrário do que se achava nas últimas décadas, cresce a compreensão de que a espasticidade parece mesmo não ser o fenômeno mais incapacitante nesses pacientes ${ }^{31,32}$.

Portanto, os dados dessa pesquisa apresentam relevantes informações para clínicos e pesquisadores no que tange a utilização de instrumentos clínicos de medida. Assim, deve-se pensar na precisa escolha dos instrumentos de medida a serem utilizados no processo de avaliação. Quanto mais fáceis, práticos e confiáveis, melhores serão as chances de estabelecer tanto o diagnóstico como um prognóstico realístico de reabilitação.

Fica a sugestão da utilização da dinamometria de preensão manual por ser um teste rápido, fácil e acessível, e que pode fazer parte dos protocolos de avaliação e predição da recuperação do membro superior de pacientes hemiparéticos por AVC.

A avaliação precisa da recuperação do membro superior permite determinar estratégias terapêuticas apropriadas e, sobretudo, discutir expectativas quanto à recuperação funcional junto ao paciente e seus familiares.

Como limitações dessa pesquisa registram-se o pequeno número de pacientes avaliados, que deve ser ampliado em pesquisas futuras; a comparação com o lado "não afetado", observando possíveis influências ipsilaterais; e o estudo comparativo dos achados encontrados com valores normativos para idade e sexo. 


\section{REFERÊNCIAS}

1. Oliveira MR, Orsini M. Escalas de avaliação da qualidade de vida em pacientes brasileiros após acidente vascular encefálico. Rev Neurocienc. 2008;17(3):255-62.

2. André C. Manual do AVC. $2^{\text {a }}$ ed. Rio de Janeiro: Revinter; 2005. 159p.

3. Costa MCF, Bezerra PP, Oliveira APR. Impacto da hemiparesia na simetria e na transferência de peso: repercussões no desempenho funcional. Rev Neurocienc. 2006;14(2):10-3.

4. Figueiredo IM, Sampaio RF, Mancini MC, Silva FCM, Souza MAP. Teste de força de preensão utilizando o dinamômetro Jamar. Acta Fisiátrica. 2007;14(2).

5. Fess EE, Moran CA. Clinical assessment recommendation. Chicago: American Society of Hand Therapists; 1981.

6. Soares AV, Suzuki S, Metzler CT. Testes preditivos para a recuperação motora do membro superior em pacientes hemiparéticos pós-AVC. Revista Científica JOPEF Online. 2008; 1:36-8.

7. Katrak P, Bowring G, Conroy P, Chilvers M, Poulos R, McNeil D. Predicting upper limb recovery after stroke: the place of early shoulder and hand movement. Arch Phys Med Rehabil. 1998;79(7):758-61.

8. Huter-Becker A, Dolken M. Fisioterapia em neurologia. $1^{\mathrm{a}}$. ed. São Paulo: Santos. 2008;98-110.

9. Gregson JM, Leathley M, Moore AP, Sharma AK, Smith TL, Watkins CL. Reliability of the tone assessment scale and the modified ashworth scale as clinical tools for assessing poststroke spasticity. Arch Phys Med Rehabil. 1999;80(9):1013-6.

10. Faria I, Salmela LFT, Michaelsen SM. Função do membro superior em hemiparéticos crônicos: análise através da classificação internacional de funcionalidade, incapacidade e saúde [dissertação]. Belo Horizonte: Escola de Educação Física, Fisioterapia e Terapia Ocupacional, Universidade Federal de Minas Gerais; 2008.

11. Mendes MF, Tilbery CP, Balsimelli S, Moreira MA, Cruz AMB. Teste de destreza manual da caixa e blocos em indivíduos normais e em pacientes com esclerose múltipla. Arq Neuropsiquiatr. 2001;59(4):889-94.

12. Lundy-Ekman L. Neurociências fundamentos para reabilitação. $3^{a}$. ed. Rio de Janeiro: Elsevier; 2008. 477p.

13. Moreira D, Álvarez RRA. Utilização dos monofilamentos de Semmes-Weisntein na avaliação de sensibilidade dos membros superiores de pacientes hansenianos atendidos no Distrito Federal. Hansen Int. 1999;24(2):121-8.

14. Araújo F, Ribeiro JLP, Oliveira A, Pinto C. Validação do índice de Barthel numa amostra de idosos não institucionalizados. Rev Port Saúde Pública. 2007;25(2):59-66.

15. Beebe JA, Lang CE. Relationships and responsiveness of six upper extremity function tests during the first six months of recovery after stroke. J Neurol Phys Ther. 2009;33(2):96-103.

16. Hendricks HT, van Limbeek J, Geurts AC, Zwarts MJ. Motor recovery after stroke: a systematic review of the literature. Arch Phys Med Rehabil. 2002;83(11):1629-37.

17. Kwakkel G, Kollen B, Twisk J. Impact of time on improvement of outcome after stroke. Stroke. 2006;37(9):2348-53.

18. Duncan PW, Goldstein LB, Matchar D, Divine GW, Feussner J. Measurement of motor recovery after stroke. Outcome assessment and sample size requirements. Stroke. 1992;23(8):1084-9.

19. Kwakkel G, Kollen BJ, van der Grond J, Prevo AJ. Probability of regaining dexterity in the flaccid upper limb: impact of severity of paresis and time since onset in acute stroke. Stroke. 2003;34(9):2181-6.

20. Beebe JA, Lang CE. Active range of motion predicts upper extremity function 3 months after stroke. Stroke. 2009;40(5):1772-9.

21. Verheyden G, Nieuwboer A, de Wit L, Thijs V, Dobbelaere J, Devos $\mathrm{H}$, et al. Time course of trunk, arm, leg, and functional recovery after ischemic stroke. Neurorehabil Neural Repair. 2008;22(2):173-9.

22. Mulder T, Nienhuis B, Paulwels J. The assessment of motor recovery: a new look at an old problem. J Electromyogr Kinesiol. 1996;6(2):137-45.

23. Wagner JM, Lang CE, Sahrmann SA, Edwards DF, Dromerick AW. Sensorimotor impairments and reaching performance in subjects with poststroke hemiparesis during the first few months of recovery. Phys Ther. 2007;87(2):751-65.

24.Smania N, Paolucci S, Tinazzi M, Borghero A, Manganotti $\mathrm{P}$, Fiaschi A, et al. Active finger extension: a simple movement predicting recovery of arm function in patients with acute stroke. Stroke. 2007;38(3):1088-90.

25.Zackowski KM, Dromerick AW, Sahrmann SA, Thach WT, Bastian AJ. How do strenght, sensation, spasticity and joint individuation relate to the reaching deficits of people with chronic hemiparesis? Brain. 2004;127 (Pt 5):1035-46.

26. Rand D, Weiss PL, Gottlieb D. Does proprioceptive loss influence recovery of the upper extremity after stroke? Neurorahabil Neural Repair. 1999;13(1):15-21. 


\section{Referências (cont.)}

27. Welmer AK, von Arbin M, Murray V, Holmqvist LW, Sommerfeld DK. Determinants of mobility and self-care in older people with stroke: importance of somatosensory and perceptual functions. Phys Ther. 2007;87(12):1633-41.

28. Winward CE, Halligan PW, Wade DT. Somatosensory recovery: a longitudinal study of the first 6 months after unilateral stroke. Disabil Rehabil. 2007;29(4):293-9.

29. Patel AT, Duncan PW, Lai SM, Studenski S. The relation between impairments and functional outcomes poststroke. Arch Phys Med Rehabil. 2000;81(10):1357-63.
30. Tilling K, Sterne JA, Rudd AG, Glass TA, Wityk RJ, Wolfe CD. A new method for predicting recovery after stroke. Stroke. 2001;32(12):2867-73.

31. Teixeira-Salmela LF, Oliveira ESG, Santana EGS, Resende GP. Fortalecimento muscular e condicionamento físico em hemiplégicos. Acta Fisiátrica. 2000;7(3):108-18.

32. O`Dwyer NJ, Ada L, Neilson PD. Spasticity and muscle contracture following stroke. Brain. 1996;119(Pt 5): 1737-49. 